\title{
Mehrsprachiger Fachsprachenaufbau im Geographieunterricht
}

\author{
*astrid.weissenburg@ph-karlsruhe.de, Institut für Transdisziplinäre Sozialwissenschaft - Geographie, Pädagogische Hochschule Karlsruhe
}

eingereicht am: 11.09.2017, akzeptiert am: 17.01.2018

\begin{abstract}
Mehrsprachigkeit ist ein gesellschaftlicher Fakt, dem sich auch Fachdidaktiken stellen müssen, da sich fachliches Vorwissen der Schüler/innen u. a. über eine hohe sprachliche Diversität auszeichnet. Es gilt sprachliche Lernvoraussetzungen nutzbar zu machen, um die Bildungschancen zu erhöhen. Der Beitrag fokussiert, ausgehend von einer theoretisch-konzeptionellen Herleitung, die Entwicklung eines systematischen, mehrsprachigen Fachkonzeptaufbaus. Dieser sprachensensible Aufbau wird an einem typischen Beispiel der Klassen 5/6 angewandt.
\end{abstract}

Keywords: Conceptual Growth, fachbezogene Bildungssprache, Fachwortschatz, Dorf, inklusive Mehrsprachigkeit

\section{Geography Education: Creating Access to Academic Language while Recognizing Plurilingualism}

Plurilingualism is a given fact. As educational subjects apply languages in subject-bound communication processes, languages are a matter of all subjects. Additionally, students apply different languages while voicing their prior knowledge. In order for students to be successful in education, plurilingual statements need to be included in educational learning processes. In the following, a theory-based approach on plurilingual conceptual development is introduced, while applying it to a common example of early secondary geography education.

Keywords: conceptual growth, subject-bound academic language, terminology, village, inclusive plurilingualism

\section{Bildungskontext sprachenbewusst wahrnehmen}

$20,3 \%$ der Bevölkerung in Deutschland weisen 2014 einen Migrationshintergrund auf. Die unter 15-Jährigen stellen in dieser Bevölkerungsgruppe wiederum einen signifikanten Anteil von 21,8\% (vgl. Statistisches Bundesamt 2016: 220). In Ergänzung zur zunehmenden räumlichen Mobilität von Personen, welche u.a. zu einer sprachlich diverseren Gesellschaft in Deutschland führt, beeinflussen die vorhandenen Dialekte - welche in den aktuellen Praktiken rückläufig sind - die sprachliche Diversität in Deutschland im besonderen Maße (vgl. Munske 2008). Diese soeben beschriebene mehrsprachige Lebenswelt prägt die Lernenden im schulpflichtigen Alter. Die von der Kultusministerkonferenz in Auftrag gegebene, nationale Studie ,Deutsch-Englisch-Schülerleistungen-International ${ }^{*}$ (vgl. Klieme 2006) weist bei Lernenden im schulpflichtigen Alter auf folgende Einflussfaktoren hin: Schüler/innen der neunten Jahrgangsstufe, die eine nicht-deutsche Erstsprache im Elternhaus sprechen und nicht mehrsprachig - ohne sprachlicher Alltagspraxis mehrerer Sprachen - aufwachsen, weisen den größten Leistungsrückstand auf (vgl. ebd.). Im Vergleich dazu weisen Jugendliche, die mehrsprachig - mit sprachlicher Alltagspraxis mehrerer Sprachen aufwachsen bereits einen geringeren Rückstand auf. Davon ausgehend, deutet die aktive Anwendung lebensweltlicher Mehrsprachigkeit, im Vergleich zur passiven Mehrsprachigkeit, auf einen Leistungszuwachs im Bildungskontext hin. Der Blick auf den Bereich des Wortschatzes ist hingegen erschreckend. Selbst in der neunten Jahrgangsstufe weisen Jugendliche nicht-deutscher Erstsprache - hierbei liegen keine nach aktiver und passiver Mehrsprachigkeit differenzierten Ergebnisse vor - im Vergleich zu deutsch-sprachigen Schülerinnen und Schülern einen erheblichen Rückstand auf (vgl. ebd.). Obwohl diese Erkenntnisse 2018 bereits mehr als zehn Jahre zurückliegen, stellt sich die Aufgaben der fachbezogenen Wortschatzbildung und -förderung im Zeitalter einer sprachlich diverser werdenden Lebenswelt der Schüler/innen umso stärker. 
Mit der neusten PISA-Untersuchung 2015 wird die Bedeutung sprachlicher Diversität in den OECDStaaten deutlich (vgl. OECD 2016). 67\% der Schüler/innen der ersten Zuwanderungsgeneration und $45 \%$ der zweiten Generation sprechen in den teilnehmenden OECD-Staaten zu Hause nicht die Sprache, in der der Test durchgeführt wird (vgl. ebd.). Die Tendenz, dass Schüler/innen im Jahr 2015 vermehrt die Herkunftssprache der Familien zu Hause sprechen und gleichzeitig weniger Vertrautheit mit der Testsprache aufweisen, deutet auf eine fehlende Verknüpfung beider (lebensweltlicher) Kommunikationsmedien hin (vgl. ebd.). Somit gilt es die sprachlich diverser werdende Schülerschaft anzuerkennen und Sprachen ressourcenorientiert im (Fach-)Unterricht einzusetzen, denn „wenn Lernende zu einem Wort bereits ein Konzept in ihrer Erstsprache haben, erleichtert dies das Lernen des deutschen Wortes" (Neugebauer \& Nodari 2014: 97). In diesem Zuge stellt die OECD (2016) die Forderung erfolgreicher und nachhaltiger Sprachförderprogramme, indem diese u. a. „die Verbindung des Erwerbs sprachlicher und fachbezogener Inhalte“ anstreben, um die Bildungschancen mehrsprachiger Schüler/innen zu stärken (OECD 2016: 299).

Aufgrund dieser lebensweltlichen Relevanz für Schüler/innen und Lehrer/innen nähert sich der Beitrag der Rolle von Sprache(n) im Bildungskontext allgemein, um anschließend eine Möglichkeit der erfolgreichen Integration von lebensweltlich vorhandenen Sprachen der Schüler/innen auf der Ebene des Fachwortschatzes im Geographieunterricht aufzuzeigen. Theoriegeleitete und argumentativ aufgebaute Schlüsse werden konzeptuell am Beispiel des Fachwortes ,Dorf konkretisiert und problematisiert. Die Entwicklung von Handreichungen für die praktische Umsetzung im Geographieunterricht ist nicht Ziel dieses Beitrags, da dieser auf der reflexiven Metaebene der Fachkonzeptentwicklung verweilt.

\section{Sprache im (geographischen) Bildungs- kontext}

Sprache stellt - ausgehend von den internationalen Testergebnissen - ein wesentliches Werkzeug zur Erreichung von Bildungsgerechtigkeit und Bildungserfolg dar. Aus einer sozialwissenschaftlichen Perspektive, die an die Soziolinguistik angelehnt ist, kann Sprache als „die Fähigkeit, abstrakte Ideen durch eine komplexe Abfolge von Zeichen oder Signalen zu kommunizieren" (Spering \& Schmidt 2009: 114) verstanden werden. Sofern die abstrakten Ideen dem Ausdruck von Erkenntnis gleichgesetzt werden, ist Sprache einerseits Ausdruck von Erkenntnis und führt andererseits zu neuer Erkenntnis, wodurch Sprache als Kontinuum verstanden werden kann. Des Weiteren dient Sprache lediglich als Referenz zur Welt, da der direkte Zugang zur Gegebenheit dem Menschen verwehrt bleibt (vgl. Felder \& Gardt 2015). Sprache als Medium des Erkenntnisprozesses zu betrachten, bestätigt die Informationsfunktion von Sprache (vgl. Adamzik 2001). Mithilfe von Sprache werden Äußerungen über einen Sachverhalt verbalisiert, welche bestimmte Bilder über den Sachverhalt vermitteln und somit zu einer Wissenskonstruktion beitragen. „Als Wissen bezeichnet man organisierte Information, die im Gedächtnis gespeichert ist" und in zwei unterschiedlichen Arten repräsentiert wird: deklaratives und prozedurales Wissen (vgl. Spering \& Schmidt 2009: 74).

Die Fokussierung des deklarativen Wissens im Erkenntnisprozess lässt den komplexen Charakter von Sprache deutlich werden. Sprache wird im mentalen Lexikon des Gehirns gespeichert und dort in mindestens zwei Formen verankert: In Anlehnung an de Saussure, besteht Sprache einerseits aus dem Wort und andererseits aus dem Konzept. Wörter sind aus Lauten bzw. Buchstaben zusammengesetzt und bilden Sprache ab. Das Konzept ist das mit dem Wort assoziierte funktionale Wissen und entspricht der begrifflichen Bedeutung (vgl. Engelkamp \& Zimmer 2006). Der im mentalen Lexikon angelegte Wortschatz wird je nach Fach präzisiert, sodass das (Fach-)Wort als kleinste sinngebende Einheit im Fachunterricht dient (vgl. Roelcke 2014). Der Fachwortschatz ist vor allem im Register der Bildungssprache, welches „besonders in Kontexten formaler Bildung relevant ist" (Petersen \& Tajmel 2015: 86), ein zentrales Element fachsprachlicher Bildung in der Schule (vgl. Gogolin \& Lange 2011).

Roelcke (2014) forciert die Wichtigkeit der fachbezogenen Terminologie, indem er postuliert, dass „[d]er Gebrauch von Fachwörtern (...) eine Tatsache [ist], an der niemand in Schule, Ausbildung und Beruf vorbeikommt. Den Gebrauch von Fachwörtern zu verweigern, würde einem Ausstieg aus weiten Teilen der Gesellschaft gleichkommen und somit Mitwirkung und Selbstbestimmung unmöglich machen" (ebd.: 93). Die sozialsymbolische Funktion von Bildungssprache ist somit ein vehementes Argument für die notwendige Verantwortungsübernahme jedes Faches den Fachwortschatzaufbau voranzutreiben (vgl. Morek \& Heller 2012).

Für das Fach Geographie hat Birkenhauer (2005) den Fachwortschatzaufbau mit Hilfe einer 5-schrittigen Einführung von Fachwörtern bestritten, an die sich die Arbeiten von Schmoll (2011) anschließen. Ziel dieser Auseinandersetzungen ist die Generierung eines Richtwertes zur maximalen Anzahl an zu lernenden Fachbegriffen für jedes Schuljahr. Im Gegensatz dazu setzen sich Kniffka \& Neuer $(2008 ; 2015)$ weniger mit der quantitativen Herangehensweise auseinander, sondern fokussieren einen systematischen 
Fachwortschatzaufbau, welcher die zuvor beschriebene Differenzierung von Wort- und Konzeptebene ins Zentrum rückt. In diesem sprachsensiblen Ansatz werden die Fachkonzepte und somit die Bedeutung der Fachwörter in den Fokus von schulischer Fachsprache (vgl. ebd. 2017) gerückt.

Ausgehend von diesem Verständnis von Sprache und der damit verbundenen Wissenskonstruktion im Register der Bildungs- und Fachsprache, obliegt die bewusste Reflexion von Sprache und dessen Einsatz aller am Bildungsprozess beteiligten Akteurinnen und Akteure.

Sprachliche Förderung als Querschnittsaufgabe aller Fächer zu verstehen (vgl. Birkenhauer 2005), indem die Reflexion von Sprache auch im Fachunterricht gefördert wird, kann im Rahmen des ,Language Awareness'-Ansatzes erfolgen. Der Ansatz zielt auf die metakognitive Fähigkeit der sprachenvernetzenden und -reflektierenden Kompetenzen (vgl. BurwitzMelzer 2003; Knapp-Potthoff 1997) und kann u.a. mithilfe von Vergleichen auf der Fachwortebene sowie über den alternierenden Einsatz unterschiedlicher Sprachen in den Unterrichtsphasen gefördert werden (vgl. Dirim \& Müller 2007). Der englische Fachbegriff der ,Awareness' umfasst das Konstrukt der Sprachbewusstheit sowie des Sprachbewusstseins und dient in seiner Komplexität einer Doppelfunktion. Im Deutschen greift das Konstrukt der Sprachbewusstheit lediglich auf die kognitive Klarheit des Sprachproblems zurück. Die Sprachbewusstheit einer Person ermöglicht über Sprachvergleiche und Reflexionen von Regeln das Sprachproblem kognitiv zu fokussieren, dieses aber in der Sprachpraxis nicht zu lösen. Das Konstrukt des Sprachbewusstseins hingegen greift auf die Produktion von Sprache und dem ,Sprachgefühl' zurück. Im Sprachverhalten der Person wird ein Problem wahrgenommen, welches jedoch ohne eine Sprachbewusstheit kognitiv nicht bearbeitet werden kann (vgl. Spitta 2000).

,Awareness 'fasst diese im Deutschen getrennten Momente des Phänomens zusammen und dient einerseits als Lernstrategie zur Reflexion sprachlicher Strukturen und auf der anderen Seite als Werkzeug der Selbstkontrolle und Kreativität (vgl. Knapp-Potthoff 1997). In Zeiten zunehmender sprachlicher Diversität wird ,Language Awareness' als bewusste Sprachpraxis zu einer unabdingbaren Kompetenz, die es in allen Schulfächern der Bildungsinstitution Schule zu fördern gilt.

Ausgehend von der bis dato aufgezeigten Komplexität gilt es die Grundannahmen zum Verständnis von Sprache im Allgemeinen (siehe Kap. 1, Absatz 1-3) sowie die sprachliche Diversität im Sinne der, Language Awareness 'im Fachwortschatzaufbau des Faches Geographie zu integrieren. Ein weiteres Argument zur Fokussierung des Fachwortschatzes, ausgehend vom Wort-Konzept-Charakter der Sprache, liegt im aktuellen, konstruktivistisch-angelegten Geographiedidaktikansatz des, Conceptual Growth' (vgl. Reinfried 2008). Denn wie bereits Duit (2008) mit Rückbezug auf Asubel (1968) hervorhebt, ist „der wichtigste Faktor, der das Lernen beeinflusst, (...) was der Lernende schon weiß. Man berücksichtige dies und lehre entsprechend" (Asubel 1968 in Duit 2008: 2). In Anlehnung an Kattmann stärkt Schuler (2013) das Modell der Didaktischen Rekonstruktion, welches in seiner Grundannahme von den Perspektiven der Schüler/ innenvorstellungen und der fachlichen Klärung ausgeht. Mit Hilfe der didaktischen Strukturierung gilt es den Schüler/innen ihre Alltagsvorstellungen bewusst zu machen und einen Vergleich mit den wissenschaftlichen Vorstellungen zu bestärken, um einen konzeptuellen Wechsel nachhaltig und anschlussfähig gestalten zu können (vgl. Duit 2008; Reinfried 2008). Der Lernzuwachs besteht in diesem Verständnis von, Conceptual Change' darin, dass sich Schüler/innen den eigenen Vorstellungen bewusst werden, um diese dann im Abgleich mit der fachwissenschaftlichen Vorstellung korrigieren zu können. Somit vollzieht die Lehrperson einen Paradigmenwechsel und die unterschiedlichen (sprachlichen) Perspektiven werden nicht erhalten.

Reinfried (2008) plädiert vor dem Hintergrund des abrupten und bewertenden Wechsels für den Begriff des, Conceptual Growth`. Die Ausgangsbasis dieses Ansatzes setzt sich ebenfalls aus den Schüler/innenvorstellungen und einer fachlichen Klärung zusammen, wobei beide Perspektiven im Sinne der, conceptual apprenticeship 'bestehenbleiben (vgl. Reinfried 2008). In diesem Verständnis geht es weniger um einen Wechsel, als vielmehr um eine kontextuelle Differenzierung von Alltags- und Fachwissen zum selben Phänomen. Schüler/innen können im Ansatz des, Conceptual Growth'zwischen Alltagskontexten und wissenschaftlichen Kontexten unterscheiden (vgl. ebd.). Ausgehend von dieser differenzierten Betrachtung des Vorwissens von Schülerinnen und Schülern im LehrLern-Prozess, wird den unterschiedlichen Sprachregistern (Alltags-, Bildungs- und Fachsprache) sowie der lebensweltlichen Mehrsprachigkeit der Schüler/innen Rechnung getragen. Schüler/innen können im Zuge des, Conceptual Growth' ihr (mehrsprachiges) Vorwissen zum Fachwort abrufen und somit den Lehr-LernProzess anschlussfähig gestalten, wie auch je nach Kontext differenzieren.

\section{Sprachsensibilisierung im Geographie- unterricht am Beispiel ,Dorf}

In Rückgriff auf die bis dato dargelegten theoretischen Überlegungen zum sprachsensiblen Aufbau und Reflexion von zeitgemäßem Geographieunterricht, gilt 
es im Folgenden die Anwendung am Beispiel des Fachwortes ,Dorf ${ }^{k}$ darzulegen. In einem aktuellen baden-württembergischen Schulbuch für den Geographieunterricht nach dem Bildungsplan 2016 wird die Siedlungsform wie folgt dargelegt: „Tatsächlich bedeutet Dorf so viel wie bäuerliche Siedlung. Typisch sind eine offene Bebauung mit einzelnen Häusern, sowie eine geringe Bevölkerungszahl und Bevölkerungsdichte" (Siegmund 2016: 120). In dieser Darlegung wird das Fachkonzept ,Dorf mit den Fachwörtern bäuerliche Siedlung, offene Bebauung sowie geringe Bevölkerungszahl und Bevölkerungsdichte belegt, sodass eine hohe Dichte an bildungssprachlicher Fachterminologie zu verzeichnen ist. Diese Komplexität steht einer angenommenen, alltagssprachlichen Schüler/innenvorstellung, die einem „ländliche[n] Ort (...) mit Bauernhöfen und Tieren" (beispielhafte Schüler/innenvorstellung aus Klasse 5) entsprechen kann, gegenüber. In der Kontrastierung dieser beiden Konzepte des Wortes ,Dorf bildet das Phänomen des Bäuerlichen die Schnittmenge. Die Äußerung der ländlichen Ortschaft weist Parallelen zur Fachterminologie der offenen Bebauung und geringen Bevölkerungszahl bzw. -dichte auf. Sofern ergänzend zur fachlichen Klärung die fachwissenschaftliche Bedeutung des Dorfes herangezogen wird, bezieht sich Dorf auf eine Siedlung des ländlichen Raumes mit linearem, polarem oder flächigem Grundriss sowie einer Regeloder Unregelmäßigkeit (vgl. Henkel 2004). Die Kontrastierung aller drei Konzepte des Fachwortes ,Dorf weisen Schnittmengen und Unterschiede auf, sodass für einen sprachensensiblen Geographieunterricht (1) die Frage nach dem wesentlichen Kern des Konzeptes ,Dorf zu stellen und (2) dieser zentrale Aspekt sprachsensibel in Anlehnung an die Lebenswelt der Schüler/ innen zu vermitteln ist. Der sprachsensible Lernprozess ist mithilfe von Entlastungsstrategien, wie dem Scaffolding, zu gestalten (vgl. Kniffka \& Roelcke 2015; Schwarze 2017). Des Weiteren können Portfolios im Fachunterricht als Reflexionsmoment inhaltlicher Aushandlungsmomente dienen (vgl. Weißenburg 2016). In Anbetracht des, Conceptual Growth' sowie mit dem Blick auf die häufig von geographischen Fachwörtern durchzogene Alltagssprache (z. B. Stadt) gilt es im sprachsensiblen Geographieunterricht diverse Konzepte des Fachwortes nebeneinander stehen zu lassen. Diese Kompetenz ist über die Thematisierung von Gemeinsamkeiten und Unterschieden im Sinne des ,Language Awareness-Ansatzes zu verfolgen. Als wesentlich ist jedoch der fachliche Kern des Konzeptes zu erachten, welchen es anschlussfähig an das Vorwissen der Schüler/innen zu vermitteln gilt. Eine stringente Aufarbeitung der Fachterminologie in aktuellen Schulbüchern ist vor diesem Hintergrund erstrebenswert.

\section{SpracheN im Geographieunterricht}

Die Annahme, dass Schüler/innen individuell differenzierte Vorstellungen mit in den Unterricht bringen, ist nicht neu (siehe Einleitung und Kap. 1). Alle Schüler/innen sprechen unter den bisherigen Annahmen jedoch dieselbe Erstsprache, nämlich die Institutionensprache Hochdeutsch. Was passiert jedoch mit der fachbezogenen Bildungssprache, wenn - wie in der Einleitung aufgezeigt - die Schüler/innenschaft sprachlich diverser wird? Wie kann die Vermittlung schulischer Fachsprache (vgl. Kniffka \& Neuer 2017) vor dem Hintergrund von inklusiver Mehrsprachigkeit (vgl. Weißenburg 2016) erfolgreich gelingen?

Die Fähigkeit Erkenntnisse durch diverse Sprachsysteme auszudrücken und neue Erkenntnisse mit Hilfe dieser sprachlichen Vielfalt zu erlangen, gehört heute zu den sogenannten, soft skills' des 21. Jahrhunderts. Der sprachlich diverse Erkenntnisprozess ist jedoch nicht nur interindividuell, sondern erfolgt in der Regel in kulturellen Praxen (vgl. Felder \& Gardt 2015). Vor dem Hintergrund dessen ist der Bildungsprozess, welcher die Anwendung mehrerer Sprachen fokussiert, auf der Konzeptebene zu reflektieren.

„Wichtig für den schulischen Aspekt ist hier vor allem (...), dass das menschliche Sprachvermögen auf Mehrsprachigkeit ausgerichtet ist, so dass individuelle und gesellschaftliche Mehrsprachigkeit den ,Normalfall' darstellen, Einsprachigkeit hingegen als Sonderfall zu verstehen ist" (Hu 2010: 71).

Mit Rückbezug auf eine ergiebige Diskussion zahlreicher Ansätze von Mehrsprachigkeit auf unterschiedlichen Maßstabsebenen (bspw. Individuum vs. Gesellschaft; Schule vs. außerschulisches Umfeld) sowie einer kritischen Analyse bildungsbezogener Ansätze zur Integration von migrationsbedingter, lebensweltlicher Mehrsprachigkeit (vgl. Gogolin 2006) konnte Weißenburg (2016) keine für den Fachunterricht angemessene theoretische Diskussion des Mehrsprachigkeitsbegriffs aufzeigen. Dennoch ist unter Einbezug bisheriger Arbeiten zur migrationsbedingten, lebensweltlichen Mehrsprachigkeit der Begriff der ,Inklusiven Mehrsprachigkeit' für das mehrsprachige Fachlernen entwickelt worden (vgl. Weißenburg 2016). Der theoriegeleitete Ansatz fokussiert die Anerkennung und Nutzung von migrationsbedingter Mehrsprachigkeit als Ressource im Fachunterricht. Inklusiv bedeutet, das Zusammendenken aller in der Lebenswelt der Schüler/innen vorhandenen Sprachen, um die Entwicklung von Fachkonzepten anschlussfähig und mit einer hohen sprachlichen Sensibilität für einen möglichen fachlichen Mehrwert voranzutreiben. Ziel des Ansatzes 
ist die theoriegeleitete und argumentativ schlüssige Herleitung einer sprachENsensiblen Perspektive für den Fachunterricht, weniger eine konkrete Handreichung einzelner Praxiselemente, da diese sich je nach gegebenem Kontext im Sinne der konstruktivistischen Geographiedidaktik verändert. Der Ansatz umfasst fünf Merkmale, welche im Folgenden skizziert werden (vgl. Weißenburg 2016):

1. Lebenswelt: Alle in der Lebenswelt vorhandenen Sprachen der Personen - hierbei sind auch Dialekte gemeint - sind aktiv einzubeziehen, sodass die lebensweltlich (migrationsbedingte) Mehrsprachigkeit (vgl. Gogolin 2000) anerkannt wird. Dies kann in Form von Kleingruppenarbeiten im Sinne der Individualisierung von Lehr-Lern-Prozessen erfolgen.

2. Ressourcenorientierung: Die Anerkennung der in der Lebenswelt vorhandenen Sprachen ist so zu gestalten, dass die einzelnen Sprachen als Wissensressource im Lernprozess angewandt werden und die Sprachen einen Nutzen erfahren (vgl. Dirim et al. 2008; vgl. Gogolin 2007). Das Wissen, welches in den Sprachen gespeichert ist, gilt es im Sinne des Wort-Konzept-Aufbaus zugänglich zu machen und aktiv in den Erwerbsprozess einzubinden. Dies kann ebenfalls in Phasen der Freiarbeit, in Partner- oder Gruppenarbeit erfolgen. Die sprachlich diversen Wissensstrukturen sollen keinesfalls separat voneinander stehen bleiben, sondern in einer Plenumsphase unter Einsatz einer Unterrichtssprache vorgestellt und diskutiert werden.

3. Individuum vs. Gesellschaft: Das Phänomen der Mehrsprachigkeit kann auf der Ebene der individuellen Person autobiographisch bewusst gemacht werden. Jedoch gilt es diese individuelle Ebene ins Verhältnis zur gesellschaftlichen Mehrsprachigkeit, welche als, Normalfall' gilt, zu setzen (vgl. Hu 2010). Die Schnittmengen und Unterschiede zwischen individueller und gesellschaftlicher Mehrsprachigkeit gilt es zu fokussieren, indem diese beschrieben, analysiert und reflektiert werden.

4. Überwindung des monolingualen Habitus: Inklusive Mehrsprachigkeit zielt auf die Überwindung des monolingualen Habitus als überholtes Konzept zur Bildung von Nationen ab. Die alleinige Förderung der Nationalsprache gilt es zu überwinden und anderen Sprachen der alltäglichen Lebenswelt eine wertschätzende Position zu ermöglichen. Sofern das Leben in einer globalisierten Welt bestritten werden muss, gilt es den monolingualen Habitus zu duellieren, nicht ihn vollständig zu verdrängen (vgl. Gogolin 2006).
Dieses Merkmal wird mit der Umsetzung der vorangehenden drei Merkmale im Unterricht simultan verfolgt.

5. Identität: Inklusive Mehrsprachigkeit zielt auf die Anerkennung und Anwendung aller in der Lebenswelt vorhandenen Sprachen der beteiligten Personen, da somit eine positive Identitätsbildung ermöglicht werden kann (vgl. Dirim 2005; Florio-Hansen \& Hu 2007). Diese positive Identitätsbildung ist ein wesentlicher Bestandteil holistischer Bildung - und somit auch sprachlicher und fachlicher Bildung. Für die praktische Umsetzung dieses Merkmals können Portfolios herangezogen werden.

Sofern ,Inklusive Mehrsprachigkeit' im Fachunterricht gefördert wird, ermöglicht „[d]ie Erlernung einer fremden Sprache (...) die Gewinnung eines neuen Standpunkts in der bisherigen Weltansicht (...)“ (Humboldt 1836 in: Felder \& Gardt 2015: 10).

Erste empirische Ergebnisse zur erfolgreichen Anwendung der zuvor dargelegten, Inklusiven Mehrsprachigkeit' im Kontext des mehrsprachigen Fachlernens im Geographieunterricht der Grundschule stellt die qualitativ-explorative Studie von Weißenburg (2016) dar. Weißenburg (2016) fokussiert in ihrer Arbeit, neben der theoriegeleiten Herleitung des mehrsprachigen Fachlernens, die Fragestellung, Welche Konzepte von mehrsprachiger Raumbildung können in den Äußerungen von 8-jährigen Schüler/innen aufgezeigt werden?'. Ziel dieser Fragestellung ist es, Momente aufzuzeigen, in denen Schüler/innen die lebensweltliche Mehrsprachigkeit als Instrument von Raumaushandlung einsetzen und Raum mit Hilfe sprachlicher Vielfalt diversifiziert wird. Der Begriff der Raumbildung greift auf den Ansatz der räumlichen Bildung nach Dickel \& Scharvogel (2013) sowie die dort rezipierte fachwissenschaftlichen Theorie zur Raumproduktion nach Lefebvre zurück. Die Raumproduktion nach Lefebvre geht von einer Trialektik räumlichen Seins aus, welche verkürzt gesprochen, eine sprachlich kodierte Aushandlung von Raum unter Berücksichtigung verschiedener (Akteurs-)Perspektiven zum Ziel hat (vgl. Rogers 2001).

Die Ausgangslage dieser Fragestellung bildet eine fünfwöchige Unterrichtssequenz, in der Drittklässler/innen einer baden-württembergischen Grundschule das Thema ,Mein Schulort' unter Einbindung aller im Klassenzimmer vorhandenen Sprachen im Sinne der Raumtheorie nach Lefebvre bestreiten. Der Korpus der erhobenen Daten setzt sich aus einer Vielzahl an Datentypen - wie Kindergruppeninterviews, Videographien, Lernportfolios - zusammen. Die wesentliche Erkenntnis der Arbeit liegt darin, dass selbst junge Schüler/innen in der Lage sind, 
Raumbildungsprozesse mehrsprachig zu durchdringen und das Konzept der Orientierung zu etablieren. Das Konzept der Orientierung am Schulort wurde über Bedingungen (wie bspw. der eigenen Erfahrung und dem Lebensweltbezug) unter Anwendung diverser Strategien (wie bspw. des Vergleichens und dem Aufbauen von Relationen in sozialen Beziehungen) schließlich durch die Benennung von Konsequenzen (wie bspw. Eigenständigkeit und die Entstehung von (Sprach-)Räumen) seitens der Schüler/innen sprachensensibel aufgebaut. Im Folgenden gilt es den Fokus auf die Kategorie ,Entstehung von (Sprach-) Räumen' der an die Grounded Theory angelehnten Analyse zu lenken, da in dieser Konsequenz die Rolle von Sprache seitens der Lernenden markant hervorgestellt wird. Die folgenden Ausschnitte dienen als Beispiele des Konstruktionsprozesses von (Sprach-) Räumen:

\section{M3: Also ich habe ja früher in Hessen gewohnt, als/l I: $/ / M h m / /$ \\ M3: //ich ja noch ganz klein war \\ I: $\quad$ Mhm/l \\ M3: //und da haben/also/ in Hessen sp/spricht man ja auch ein bisschen anders, als in Deutschland.// (KG_5_1148-1157)}

SS15: Du weisst schon, dass niemand dir zuhört (lacht).

YS10: Nääähh und dir auch nicht.

SS15: Hallo, ich sag doch gar nix.

YS10: Doch, siehste. (...) Ben simdi türkce konusuyorum, Pech.

Wohingegen M3 im ersten Beispiel den sprachlichen Unterschied zwischen dem Hochdeutschen und dem Hessischen in ihrer Aussage während des Interviews reflektiert, setzt YS10 im Klassengespräch mit der/dem Mitschüler/in SS15 Momente der sprachlichen Exklusion ein, indem ein Sprachwechsel vollzogen wird. Beide Beispiele sind Hinweise darauf, dass mit Hilfe von SpracheN Räume konstituiert werden. Sofern weitere Beispiele zur ,Entstehung von (Sprach-) Räumen' herangezogen werden (vgl. Weißenburg 2016), kann Sprache als Machtinstrument in der Aushandlung räumlicher Bildung im Konzept der Orientierung von Schüler/innen wahrgenommen werden. Diese kurze Sequenz dient an dieser Stelle lediglich zur Veranschaulichung der sprachlichen und konzeptuellen Komplexität von räumlichen Aushandlungsprozessen in einer dritten Grundschulklasse. Schüler/ innen sind bereits im jungen Alter in der Lage, sprachensensible Konzeptbildungsprozesse erfolgreich zu gestalten, auch wenn diese eine hohe Komplexität aufweisen (vgl. ebd.).

\section{SprachENsensibilisierung im Geographie- unterricht am Beispiel ,Dorf}

Ausgehend von dem in Kap. 3 gewährten kurzen, empirischen Einblick in den mehrsprachigen geographischen Bildungsprozess von Grundschüler/innen gilt es nun, die in Kap. 2 aufgezeigte Sprachsensibilisierung im Geographieunterricht um die Dimension der ,Inklusiven Mehrsprachigkeit' nach Weißenburg (2016) zu erweitern. Wie kann das Konzept des ,Dorfs' nun sprachENsensibel gestaltet werden?

In dem zuvor eröffneten Beispiel ist auf der Ebene des alltagssprachlichen Registers zum Konzept ,Dorf lediglich vom deutschsprachigen Alltagskonzept die Rede. Sofern laut Schuler (2013) den alltagssprachlichen Schüler/innenvorstellungen Bedeutung im Lernprozess beigemessen wird, weist Konerding (2015) darauf hin, dass „der dazugehörige (deklarative) Wissenserwerb eng mit dem Erstsprachenerwerb verknüpft (ist)“ (ebd.: 67). Die erworbene Erstsprache stellt in der Aktivierung alltagssprachlicher Konzepte eine signifikante Rolle dar, denn „wenn Lernende zu einem Wort bereits ein Konzept in ihrer Erstsprache haben, erleichtert dies das Lernen des deutschen Wortes" (Neugebauer \& Nodari 2014: 97). Unter Berücksichtigung dieser Erkenntnisse sowie der empirischen Einblicke in Weißenburg (2016) gilt es alle lebensweltlichen Sprachen für die Entwicklung des bildungssprachlichen Fachwortschatzes wahrzunehmen und als Ressource einzusetzen.

Sofern beispielsweise die Erstsprache Russisch der Schüler/innen eingebunden wird, erfährt das Konzept des Dorfes eine bisher, im Deutschen unbeachtete Differenzierung: Das Wort село́ umschreibt im Russischen ein Dorf ohne Kirche, wohingegen das Wort дере́вня ein Dorf mit Kirche beschreibt. Diese fachsprachliche Differenzierung, welche die russische Sprache vornimmt, wird im Deutschen nicht vollzogen. Das geographische Fachwort ,Dorf erfährt durch die Reflexion der sprachlichen Vielfalt eine konzeptuelle Diversifikation, welche die kognitiven Strukturen im mentalen Lexikon vernetzt. Schüler/innen, die aufgrund eines sprachensensiblen Fachwortvergleichs das Unterscheidungskriterium ,Kirche' beim Fachwort ,Dorf erfahren, können somit ihr Konzept ,Dorf ${ }^{x}$ um ein weiteres Kriterium vervielfältigen. Die monolinguale, deutsche Thematisierung des Fachwortes ,Dorf ${ }^{\natural}$ würde diese sprachlich-konzeptuelle Vielfalt missachten, den Mitschüler/innen diesen Erkenntnisprozess vorenthalten und mehrsprachig russischen Kindern eine Verknüpfung im Sinne des, ConceptualGrowth-Ansatzes' verwehren. Die ressourcenorientierte Einbindung der lebensweltlich vorhandenen Sprachen ermöglicht in diesem Beispiel die Erweiterung des Fachkonzepts Dorf und stellt für das Fach 
Geographie eine wesentliche Wissenserweiterung dar. Indem Schüler/innen ihre fachlichen Wissensstrukturen vervielfältigen und vernetzen, können sie diese im Sinne der ganzheitlichen Bildung in anderen Kontexten flexibler anwenden.

Unter aktiver Einbeziehung inklusiver Mehrsprachigkeit in den Aufbau schulischer Fachsprache (vgl. Kniffka \& Neuer 2017) erfahren Schüler/innen (1) die Anerkennung ihrer lebensweltlich verankerten Ressource im institutionalisierten Lernprozess, (2) die Ausbildung von language awareness über die Arbeit mit kontrastiven Konzepten und (3) eine fachlich bereichernde Differenzierung des Fachkonzepts ,Dorf, die im monolingualen Habitus verwehrt geworden wäre. Unter Berücksichtigung dieser Stärken mehrsprachiger Bildungssprache gilt es den Aufbau des Fachwortschatzes sprachensensibel im Sinne der Wort-Konzept-Systematik zu erforschen und langfristig zu etablieren, sodass der Prozess des, Conceptual Growth' für alle Schüler/innen erfahrbar gemacht wird - und nicht Schüler/innen mit einer anderen Erstsprache am deklarativen Wissensaufbau gehindert, wenn nicht sogar ausgegrenzt werden.

\section{Zukunftsperspektive: Sprachliche Diversität im Geographieunterricht reflektieren}

Rückblickend auf die verfolgte Diskussion zum Stellenwert von Sprache im Prozess des (deklarativen) Wissenserwerbs im allgemeinen Bildungskontext sowie konkret im Geographieunterricht, gilt es einen fachsystematischen Begriffsaufbau unter Berücksichtigung von alltags-, bildungs- und fachsprachlichen Kompetenzen einer sprachlich diversen Schüler/innenschaft zu verfolgen.

Möglichkeiten der kritischen Auseinandersetzung mit einer sprachENsensiblen Fachdidaktik sind daher auf mehreren Ebene zu vollziehen:

(a) Das Konzept der Language Awareness gilt es fachübergreifend zu fördern, da sich die Mehrheit der am Bildungsprozess beteiligten Fächer über das Kommunikationsmedium der Sprache ausdrücken. Sprache ist und kann nicht alleinige Aufgabe der Sprachwissenschaften sein, wenn Individuen sich ihrer dauerhaft im Bildungsprozess bedienen. Diese Vernetzung ist in Theorie, Forschung und Praxis voranzutreiben. Eine Möglichkeit dieser Kompetenzförderung in der Praxis liegt in der Führung von Fachportfolios mit sprachsensiblen Anteilen (vgl. Weißenburg 2016). Als Beispiel ist hier zu nennen, dass vor der Unterrichtssequenz das Vorwissen zum zentralen Fachwort ,Dorf festgehalten werden kann. Dies kann sprachlich frei erfolgen. Der Lernprozess kann bspw. durch gezielte Fragen wie, Was hast du heute zum Thema ,Dorf gelernt?' im Rahmen des Portfolios begleitet werden, bevor am Ende eine eigene Definition niedergeschrieben werden kann. Der gesamte Prozess kann mehrsprachig gestaltet werden.

(b) Sprachliche Diversität ist im Jahr 2018 schulischer Fakt. Lehrer/innen brauchen dringend Unterstützung in der Praxis, sodass Unterstützungsmaßnahmen für Fachlehrkräfte im aktiven Dienst entwickelt werden müssen. Fachlehrkräfte sind keine sprachwissenschaftlich ausgebildeten Lehrkräfte, und können dennoch einen erheblichen Beitrag zur sprachlichen Sensibilisierung und zum Prozess des, Conceptual Growth 'beitragen.

(c) Angehende Lehrer/innen müssen frühzeitig in der ersten Phase der Lehrer(aus)bildung für das Thema sensibilisiert werden. Dies kann durch die Mitarbeit an aktuellen Forschungsprojekten sowie durch erste eigene Praxisübungen in den Schulpraktika angeleitet und reflektiert werden.

\section{Literatur}

Adamzik, K. (2001): Sprache: Wege zum Verstehen. Tübingen \& Basel: A. Francke Verl.

Birkenhauer, J. (2005): Sprache und Begrifflichkeit im Geographieunterricht. In: Praxis Geographie 1, 42-43.

Burwitz-Melzer, E. (2003): Neue curriculare und methodische Anforderungen an den Englischunterricht im Kontext der Mehrsprachigkeit (Kl. 1-10). In: Meißner, F.-J. \& I. Picaper (Hrsg.): Mehrsprachigkeitsdidaktik zwischen Frankreich, Belgien und Deutschland. La didactique du plurilinguisme entre la France, la Belgique et l'Allemagne, 20-31.

Dickel, M. \& Scharvogel, M. (2013): Räumliches Denken im Geographieunterricht. In: Kanwischer, D. (Hrsg.): Geographiedidaktik. Ein Arbeitsbuch zur Gestaltung des Geographieunterrrichts. Stuttgart: Borntraeger, 57-68.

Dirim, I. (2005). Notwendig ist die Schaffung einer Wissensbasis für den Umgang mit Mehrsprachigkeit. In Gogolin, I., U. Neumann, H.-J. Roth (Hrsg.): Sprachdiagnostik bei Kindern und Jugendlichen mit Migrationshintergrund, 81-86.

Dirim, İ. \& A. Müller (2007): Sprachliche Heterogenität. Deutsch lernen in mehrsprachigen Kontexten. In: Praxis Deutsch. Zeitschrift für den Deutschunterricht 34/202, 6-14.

Dirim, I., K. Hauenschild, B. Lütje-Klose, J.M. Löser \& I. Sievers (Hrsg.) (2008): Ethnische Vielfalt und Mehrsprachigkeit an Schulen. Beispiele aus verschiedenen nationalen Kontexten. Frankfurt am Main: Brandes \& Apsel.

Duit, R. (2008): Zur Rolle von Schülervorstellungen im Unterricht. In: geographie heute 29/265, 2-6.

Engelkamp, J. \& H.D. Zimmer (2006): Lehrbuch der Kognitiven Psychologie. Göttingen: Hogrefe Verl. 
Felder, E. \& A. Gardt (Hrsg., 2015): Handbuch Sprache und Wissen. Handbücher Sprachwissen. Berlin \& Boston: De Gruyter.

Florio-Hansen, I. de \& A. Hu (Hrsg.) (2007): Plurilingualität und Identität. Zur Selbst- und Fremdwahrnehmung mehrsprachiger Menschen. Tübingen: Stauffenburg Verl.

Gogolin, I. (2000): Erziehungswissenschaftliche Reflexion über den Widerspruch von „monolingualem!“ Unterricht und „multilingualer“ Realität. In: Böhme, P.,R. Heil, F. Kessl, S. Landhäußer \&T. Reinke (Hrsg.): Von der Notwendigkeit der Erziehungswissenschaft, 117-126.

Gogolin, I. (2006): Mehrsprachigkeit und die Chance auf Bildungserfolg. Über Ansprüche an das Lehren von Sprache, nicht nur im Deutschunterricht. In: Krumm, H.-J. \&P.R. Portmann (Hrsg.): Begegnungssprache Deutsch - Motivation, Herausforderung, Perspektiven: Schwerpunkt: Sprachenpolitik und fachbezogene Grundsatzfragen, 95-106.

Gogolin, I. (2007): „Das ist doch kein gutes Deutsch“Über Vorstellungen von „guter" Sprache und ihren Einfluss auf Mehrsprachigkeit. In: Florio-Hansen, I. de \& A. $\mathrm{Hu}$ (Hrsg.): Plurilingualität und Identität. Zur Selbst- und Fremdwahrnehmung mehrsprachiger Menschen, 59-69.

Gogolin, I. \& I. Lange (2011): Bildungssprache und durchgängige Sprachbildung. In: Fürstenau, S. \& M. Gomolla (Hrsg.): Migration und schulischer Wandel: Mehrsprachigkeit, 107-129.

Henkel, G. (2004): Der Ländliche Raum. Gegenwart und Wandlungsprozesse seit dem 19. Jahrhundert in Deutschland. 4. ergänzte und neu bearbeitete Auflage. Studienbücher der Geographie. Stuttgart: Borntraeger.

Hu, A. (2010): Migrationsbedingte Mehrsprachigkeit und schulischer Fremdsprachenunterricht - revisited. In: Appel, J., S. Doff, J. Rymarczyk \& E. Thaler (Hrsg.): Foreign language teaching - history, theory, methods, 65-82.

Klieme, E. (2006). Zusammenfassung zentraler Ergebnisse der DESI-Studie. Frankfurt am Main: Deutsches Institut für Internationale Pädagogische Forschung.https:// www.dipf.de/de/forschung/aktuelle-projekte/pdf/biqua/ DESI_Ausgewaehlte_Ergebnisse.pdf (03.01.2018)

Knapp-Potthoff, A. (1997): Sprach(lern)bewußtheit im Kontext. In: Fremdsprachen Lehren und Lernen (FLuL) 26, 9-23.

Kniffka, G. \& B. Neuer (2008): „Wo geht's hier nach Aldi?“ - Fachsprachen lernen im kulturell heterogenen Klassenzimmer. In: Budke, A. (Hrsg.): Interkulturelles Lernen im Geographieunterricht, 121-135.

Kniffka, G. \& B. Neuer (2015): Fachwortschatz in Geographie-Lehrwerken. In: Mitteilungen des Deutschen Germanistenverbandes 624, 346-362.

Kniffka, G.\& B. Neuer (2017): Sprachliche Anforderungen in der Schule. In: Günther, H.,G. Kniffka, G. Knoop \& T. RieckeBaulecke (Hrsg.): Basiswissen Lehrerbildung: DaZ, 38-50.

Kniffka, G. \& T. Roelcke (2015): Fachsprachenvermittlung im Unterricht. Paderborn: Schöningh.

Konerding, K.-P. (2015): Sprache und Wissen. In: Felder, E. \& A. Gardt (Hrsg.): Handbuch Sprache und Wissen. Handbücher Sprachwissen, 57-80.
Morek, M. \& V. Heller (2012). Bildungssprache - Kommunikative, epistemische, soziale und interaktive Aspekte ihres Gebrauchs. In: Zeitschrift für Angewandte Linguistik (ZfAL) 57, 67-101.

Munske, H. H. (Hrsg., 2007): Sterben die Dialekte aus? Vorträge am Interdisziplinären Zentrum für Dialektforschung an der Friedrich-Alexander-Universität Erlangen-Nürnberg, 22.10.-10.12.2007. https://www. dialektforschung.phil.uni-erlangen.de/publikationen/ sterben-die-dialekte-aus.shtml (03.01.2018).

Neugebauer, C. \& C. Nodari (2014): Förderung der Schulsprache in allen Fächern. Praxisverschläge für Schulen in einem mehrsprachigen Umfeld. Kindergarten bis Sekundarstufe I. Impulse zur Unterrichtsentwicklung. Bern: Schulverlag plus AG.

OECD (2016): PISA 2015 Ergebnisse (Band I). Exzellenz und Chancengerechtigkeit in der Bildung. PISA. Bielefeld: Bertelsmann.

Petersen, I. \& T. Tajmel (2015): Bildungssprache als Lernmedium und Lernziel des Fachunterrichts. In: Leiprecht, R. \& A. Steinbach (Hrsg.): Schule in der Migrationsgesellschaft. Ein Handbuch. Band 2: Sprache - Rassismus - Professionalität, 84-111.

Schmoll, L. (2011): Zum Umgang mit Fachsprache im Geographieunterricht. In: Praxis Geographie 9, 56-57.

Schuler, S. (2013): Geographielernende - Interesse und Vorwissen. In: Kanwischer, D. (Hrsg.): Geographiedidaktik. Ein Arbeitsbuch zur Gestaltung des Geographieunterrichts, 81-91.

Schwarze, S. (2017): Fachsprachliche Bildung in der Geographie. In: GW-Unterricht 148/4, 16-25.

Siegmund, A. (Hrsg.) (2016): Diercke Geographie 5/6. Gymnasium Baden-Württemberg. Braunschweig: Westermann.

Spering, M. \& T. Schmidt (Hrsg., 2009): Allgemeine Psychologie kompakt. Wahrnehmung, Aufmerksamkeit, Denken, Sprache. Mit Online-Materialien. Weinheim \& Basel: Beltz PVU.

Spitta, G. (2000): Sind Sprachbewusstheit und Sprachbewusstsein dasselbe? Oder Gedanken zu einer vernachlässigten Differenzierung. https://elib.suub.uni-bremen.de/ edocs/00101144-1.PDF (03.01.2018).

Statistisches Bundesamt (Hrsg., 2016): Datenreport 2016. Ein Sozialbericht für die Bundesrepublik Deutschland. Reihe Zeitbilder. Bonn: Bundeszentrale für politische Bildung.

Reinfried, S. (2008): Schülervorstellungen und Lernen von Geographie. In: geographie heute 29/265, 8-13.

Roelcke, T. (2014): Schülerinnen und Schüler brauchen Fachwörter! Ein Plädoyer. In: Der Deutschunterricht 665, 90-95.

Rogers, T.B. (2002): Henri Lefebvre, Space and Folklore. In: Ethnologies 24/1, 21-44.

Weißenburg, A. (2016): Plurilingual Approaches to Spatial Education - Perspectives of Primary Schools in the German Context. Dissertation 07.01.2016. http://hdl.handle.net/10993/26858 (03.01.2018). 